\title{
Optical control of the refractive index of a single atom
}

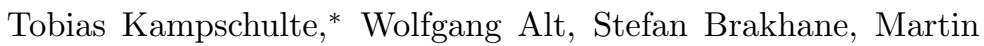 \\ Eckstein, René Reimann, Artur Widera, and Dieter Meschede \\ Institut für Angewandte Physik, Universität Bonn, Wegelerstraße 8, 53115 Bonn, Germany
}

\begin{abstract}
We experimentally demonstrate the elementary case of electromagnetically induced transparency (EIT) with a single atom inside an optical cavity probed by a weak field. We observe the modification of the dispersive and absorptive properties of the atom by changing the frequency of a control light field. Moreover, a strong cooling effect has been observed at two-photon resonance, increasing the storage time of our atoms twenty-fold to about 16 seconds. Our result points towards all-optical switching with single photons.
\end{abstract}

The properties of an optically probed atomic medium can be changed dramatically by the coherent interaction with a near-resonant control light field. The simultaneous interaction with two light fields gives rise to intriguing phenomena, such as EIT [1, 2, leading for example to the propagation of slow light $3 \sqrt{6}$. The mixed states of light and matter formed in this case can be interpreted as polaritons 7, originally introduced in the many-particle limit of solid state physics 8 . This concept is illustrated by the storage of light in and retrieval from an atomic ensemble [7, 9 11]. At the single-particle level, the control of optical properties can be utilized for atom-light quantum interfaces [12] or quantum gates [13. Coherent population trapping or EIT in systems like single ions in free space [14] or superconducting artificial atoms [15, 16, have recently been observed. With atoms, one usually requires large ensembles that are optically thick for the probe field in order to obtain strong effects. The coupling to a high-finesse cavity can be utilized to observe strong EIT signals also with single particles. Whereas this has been achieved in the resonant regime [17, we study the off-resonant case, where we are sensitive to both absorptive and dispersive effects and observe significant cooling.

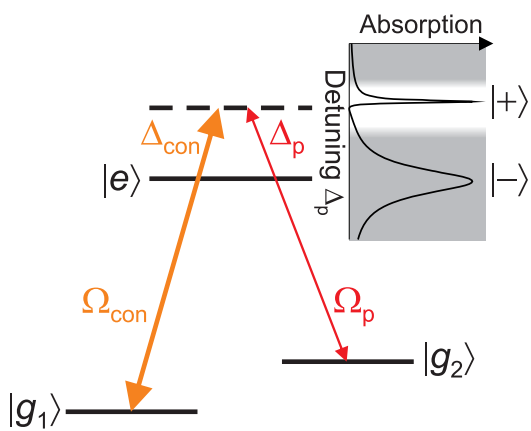

FIG. 1. Energy level scheme of a three-level atom in free space, interacting with a strong control laser with Rabi frequency $\Omega_{\text {con }}$ detuned by $\Delta_{\text {con }}$ from the atomic $\left|g_{1}\right\rangle \leftrightarrow|e\rangle$ transition. The inset shows the absorption of a weak probe beam with Rabi frequency $\Omega_{\mathrm{p}}$ as a function of its detuning $\Delta_{\mathrm{p}}$ from the atomic $\left|g_{2}\right\rangle \leftrightarrow|e\rangle$ transition, highlighting the region around the state $|+\rangle$, relevant for our experiment. In our measurement, we use a cavity field as a probe field.
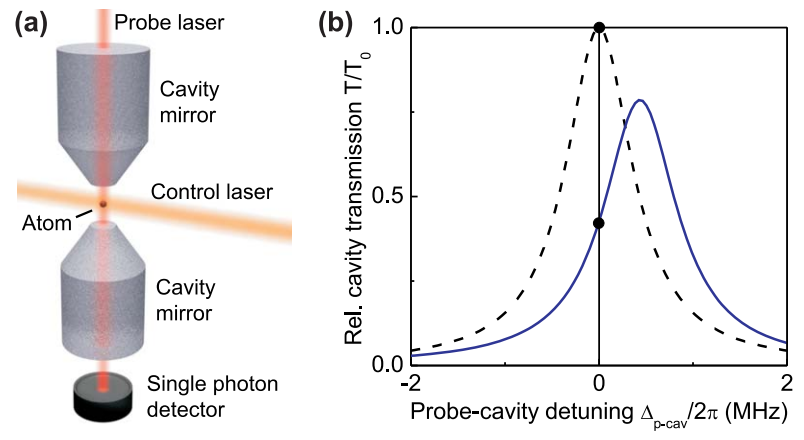

FIG. 2. (a) Schematic experimental setup. A single atom is placed between the mirrors of a high-finesse cavity and illuminated by a control laser. The transmission of a weak probe laser is detected by a single photon counting module. (b) Calculated relative cavity transmission $T / T_{0}$ for zero (dashed line) and one atom in state $\left|g_{2}\right\rangle$ (coupling strength: $g / 2 \pi=3 \mathrm{MHz}$; no EIT) inside the cavity (solid line) as a function of the detuning $\Delta_{\text {p-cav }}$ of the probe laser from the resonance frequency of the empty cavity. The dispersion and absorption by the atom lead to a shift of the cavity resonance and a reduction of the maximum transmission, respectively.

The optical control mechanism is based on an interference phenomenon: In a medium with three internal states (Fig. 1), a control field $\Omega_{\text {con }}$ couples a short-lived excited state $|e\rangle$ to a long-lived ground state $\left|g_{1}\right\rangle$. Two new eigenstates $| \pm\rangle$, so called dressed states, are formed, represented by the two absorption peaks. At two-photon resonance $\left(\delta=\Delta_{\mathrm{p}}-\Delta_{\text {con }}=0\right)$, where $\Delta_{\mathrm{p}}$ and $\Delta_{\text {con }}$ are the detunings of probe and control laser field from the atomic transitions, the medium becomes transparent due to a destructive interference between the excitation pathways to the states $|+\rangle$ and $|-\rangle$. In the case of large detunings $\Delta_{\mathrm{p}}, \Delta_{\text {con }}$ compared to the natural line width $\gamma$, the absorption peaks become strongly asymmetric due to their very different contributions from states $\left|g_{1}\right\rangle$ and $|e\rangle$. Here, we investigate a small region (highlighted in Fig. 1) containing both the narrow state $|+\rangle$ and the transparency point, where both absorption and dispersion change rapidly with $\delta$, providing a powerful tool to control the optical properties of an atom.

In our experiment we use a single or up to three laser- 

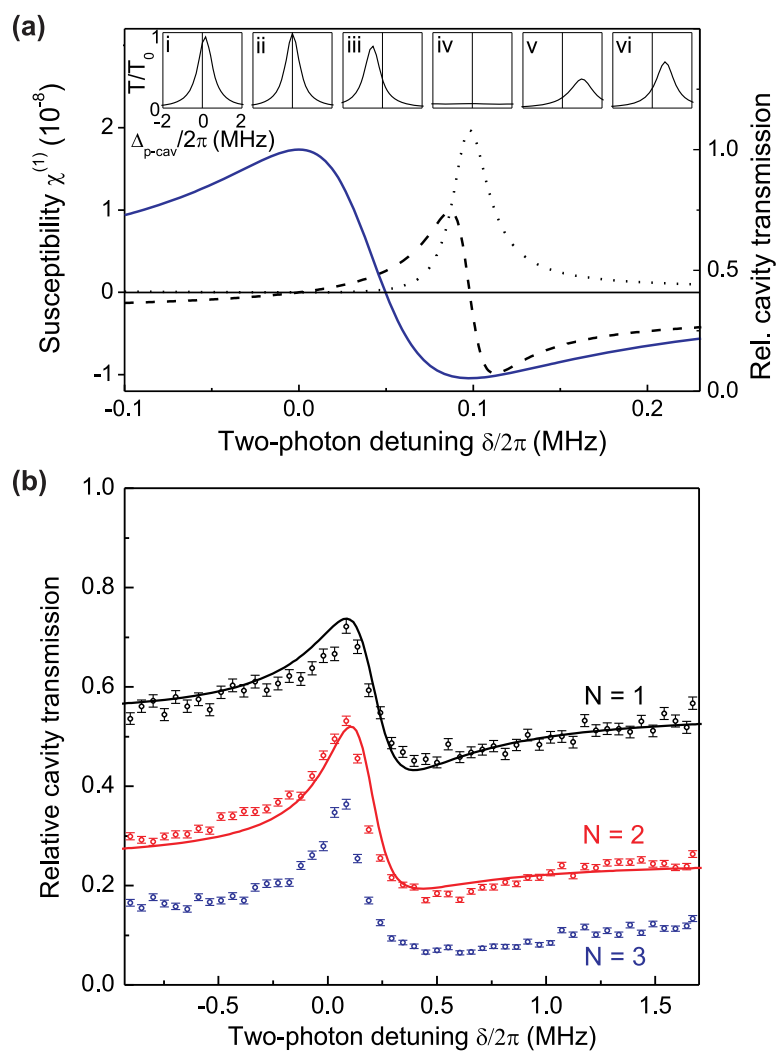

FIG. 3. (a) Real part (dashed line) and imaginary part (dotted line) of the linear susceptibility $\chi^{(1)}$ of a three-level atom inside the mode volume of a cavity as a function of the twophoton detuning $\delta$ with $\left(\Omega_{\text {con }}, \Delta_{\mathrm{p}}\right) / 2 \pi=(2.8,20) \mathrm{MHz}$. The dotted line corresponds to the absorption inside the highlighted region in Fig. 1. The solid line shows the relative probe laser transmission for a single atom in the weak probing limit without dephasing, obtained from a numerical solution of the master equation with $g / 2 \pi=3 \mathrm{MHz}$. The small insets (i-vi) are calculated cavity transmission functions as in Fig. 2 (b) for a series of fixed values of $\delta$, i.e. fixed values of $\chi^{(1)}(\delta)$, with $-50 \mathrm{kHz} \leq \delta / 2 \pi \leq 200 \mathrm{kHz}$. (b) Relative transmission of the probe laser through the cavity as a function of the twophoton detuning $\delta$, for $N=1,2,3$ atoms. The experimental data are shown as open circles with statistical error. The solid lines show numerical solutions of the master equation, taking AC-Stark shifts, dephasing, and the nonvanishing probe laser power into account.

cooled cesium atoms where the relevant states are the hyperfine ground states $\left|g_{1,2}\right\rangle=\left|6^{2} \mathrm{~S}_{1 / 2}, F=3,4\right\rangle$ and the excited state $|e\rangle=\left|6^{2} \mathrm{P}_{3 / 2}, F=4\right\rangle$ of the $\mathrm{D}_{2}$-transition at $852 \mathrm{~nm}$. We position our atoms into a high-finesse cavity $\left(\mathcal{F}=1.2 \cdot 10^{6}\right)$ using an optical conveyor belt at $1030 \mathrm{~nm}$, which also serves as a permanent trap for the atoms (for details see [18]). Atoms in state $\left|g_{2}\right\rangle$ are coupled to the resonator field with a coupling strength $g / 2 \pi=0 \ldots 12 \mathrm{MHz}$, depending on their Zeeman substate $\left|m_{F}\right|=0, \ldots, 4$. The decay rates of the cavity field and the atomic dipole are $\kappa / 2 \pi=0.4 \mathrm{MHz}$ and $\gamma / 2 \pi=2.6 \mathrm{MHz}$, respectively. For strong coupling, a photon has a high probability to be either absorbed or experience a significant phase shift already by a single atom. To change the optical properties of the atoms, we irradiate them with a control laser beam with a typical power of $1 \mu \mathrm{W}$ and a beam diameter of $170 \mu \mathrm{m}$ propagating orthogonal to the cavity field, see Fig. 2(a). A probe laser beam, resonant with the cavity, populates the empty cavity with on average $n_{\mathrm{p}}=0.1$ photons, and the transmitted ones are detected by a single photon counting module. In order to be sensitive to both absorptive and dispersive effects and to obtain a stable atom-cavity coupling, we blue-detune both the probe laser and the cavity by $\Delta_{\mathrm{p}} / 2 \pi=20 \mathrm{MHz}$ from the atomic $\left|g_{2}\right\rangle \leftrightarrow|e\rangle$ transition. Thereby, we simultaneously use the cavity as a signal amplifier and as a phase-to-amplitude converter, see Fig. 2(b): The dispersive effect of a single atom in state $\left|g_{2}\right\rangle$ shifts the cavity resonance, while the absorption directly reduces the overall amplitude, such that the transmission of the probe, now situated on the steep slope, drops to about $50 \%$ of the empty cavity level.

We record the cavity transmission while sweeping the two-photon detuning within $10 \mathrm{~ms}$ from $\delta=-0.9 \mathrm{MHz}$ to $1.7 \mathrm{MHz}$ back and forth after $N=1,2,3$ atoms have been placed into the cavity, see Fig. 3(b). We always keep the probe-cavity detuning $\Delta_{\text {p-cav }}=0$, different from normal-mode scans [17, and scan $\Delta_{\text {con }}$ instead. We alternate each sweep with a $10 \mathrm{~ms}$ cooling interval by setting $\delta=0$ (see below). After 20 measurement and cooling intervals, we switch the control beam off and a repumper on, resonant with the $\left|g_{1}\right\rangle \leftrightarrow|e\rangle$ transition, and record the cavity transmission for another $20 \mathrm{~ms}$. Then, the atoms are retrieved from the cavity and counted again. We post-select those data traces in which no atom has been lost and all atoms have coupled strongly to the cavity mode: We first check if the relative cavity transmission at the end of each sequence is below $70 \%$. For more than one atom, we also analyze a fluorescence image 19. taken at the beginning of each sequence and check if the atoms are well within the cavity mode, i.e. that their average position-dependent coupling is at least $95 \%$ of the maximum value. Each data trace shown in Fig. 3(b) is an average of about 100 single sequences. We observe a strong dispersive EIT signal close to the two-photon resonance: At $\delta \approx 0$, we find a transmission maximum followed by a minimum about $250 \mathrm{kHz}$ away.

This shape of the cavity transmission signal, which is determined by both absorption and dispersion of the atoms, can be qualitatively understood with the help of a semiclassical model [2]: Fig. 3(a) shows the real (dispersive) and imaginary (absorptive) part of the linear susceptibility $\chi^{(1)}$ of an atom with three internal states, off-resonantly interacting with a strong control and a weak probe field. For the case of one atom inside an optical cavity, the calculated transmission in the limit of weak probing is shown as a solid line. Here we consider the atom coupled with $g / 2 \pi=3.0 \mathrm{MHz}$ to the cav- 
(a)

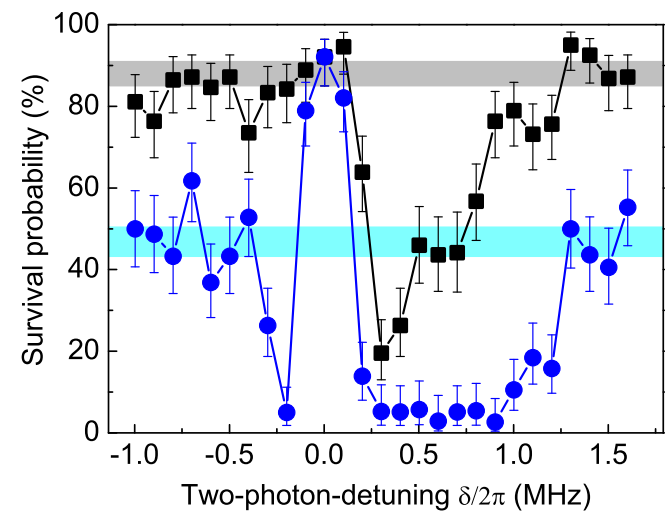

(b)

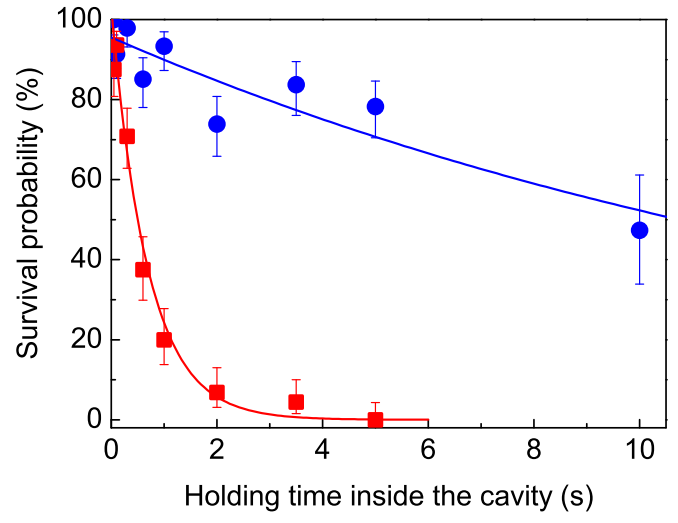

FIG. 4. (a) Probability of one atom to remain trapped inside the cavity (survival probability) as a function of the twophoton detuning $\delta$ for a holding time of $20 \mathrm{~ms}$ (squares) and $300 \mathrm{~ms}$ (circles). The shaded regions indicate the corresponding probabilities (top to bottom) for large two-photon detunings. (b) Survival probability of single atoms inside the cavity as a function of time. The circles show the survival probability with the control laser at two-photon resonance and the squares show the survival with the far-detuned trapping lasers switched on only. The error bars in both graphs are statistical.

ity mode and the Rabi frequency of the control field of $\Omega_{\text {con }} / 2 \pi=2.8 \mathrm{MHz}$, as obtained from the model (see below).

Depending on the two-photon detuning $\delta$, we identify three different regimes:

At two-photon resonance $\chi^{(1)}$ vanishes in the ideal case without dephasing [Fig. 3(a), region ii], thus the medium becomes transparent and the refractive index takes the vacuum value $n=\left(1+\chi^{(1)}\right)^{1 / 2}=1$. This leads to a cavity transmission equal to the empty cavity case.

At $\delta_{\mathrm{abs}} \approx \Omega_{\text {con }}^{2} /\left(4 \Delta_{\text {con }}\right) \approx 2 \pi \times 0.1 \mathrm{MHz}$ (iv), the model shows an absorption peak. Here, the increased losses from the resonator due to spontaneous scattering of the probe laser field by the atom lead to a transmission minimum. The full width at half maximum of the absorption peak $\Delta \delta_{\text {abs }} \approx \gamma \Omega_{\text {con }}^{2} /\left(2 \Delta_{\text {con }}^{2}\right) \approx 2 \pi \times 25 \mathrm{kHz}$ is much less than the atomic line width $\gamma$, illustrating the large ground state contribution to the dressed state $|+\rangle=\left(1-\epsilon^{2}\right)^{1 / 2}\left|g_{1}\right\rangle+\epsilon|e\rangle$, with $\epsilon \approx \Omega_{\text {con }} /\left(2 \Delta_{\text {con }}\right) \ll 1$ 20.

In the other regions (i, iii, v, vi), dispersion dominates over absorption. Here, the change in transmission is caused mainly by the shift of the cavity resonance frequency, tuning the cavity out of resonance with the probe laser. The sign of dispersion changes twice, at $\delta=0$ and $\delta=\delta_{\text {abs. }}$. In the regions $(\mathrm{i}, \mathrm{v}, \mathrm{vi})$ with negative dispersion and thus $\operatorname{Re}(n)<1$, the cavity resonance is shifted to larger frequencies, and vice versa. Finally, in the limit of large two-photon detunings $|\delta| \gg \delta_{\text {abs }}$, the control laser essentially acts as an incoherent repumping laser, which pumps atoms that are in state $\left|g_{1}\right\rangle$ to state $\left|g_{2}\right\rangle$, and the transmission approaches the value for a single atom in $\left|g_{2}\right\rangle$, as in Fig. 2(b).

For our experimental system, one has to take into account the deviation from the weak-probing limit due to the nonzero probe laser power (in a classical picture: $\left.\Omega_{\mathrm{p}}=2 g \sqrt{n_{\mathrm{p}}} \leq 2 \pi \times 2 \mathrm{MHz} \approx \Omega_{\text {con }}\right)$, as well as the contributions of other excited hyperfine states. In addition, ground state dephasing, caused by spatial and temporal inhomogeneities, e.g. fluctuating atom-cavity coupling, inhomogeneous light shifts and residual magnetic fields has been included in a more realistic model. The phenomenological ground state dephasing rate $\gamma_{\text {deph }}$ sets a lower limit for the width of the slope of the EIT signal and reduces the maximum transmission and increases the minimum transmission. We calculate the cavity transmission as a function of the two-photon detuning $\delta$ by finding the steady-state density matrix. We approximate the system by $N$ atomic five-level systems with two ground states $\left|g_{1,2}\right\rangle=\left|6^{2} \mathrm{~S}_{1 / 2}, F=3,4\right\rangle$ and three excited states $|d\rangle,|e\rangle,|f\rangle=\left|6^{2} \mathrm{P}_{3 / 2}, F=3,4,5\right\rangle$. The ground state $\left|g_{2}\right\rangle$ is coupled to all three excited states via the cavity field, and ground state $\left|g_{1}\right\rangle$ is coupled to $|d\rangle$ and $|e\rangle$ via the control laser $\left(\left|g_{1}\right\rangle \leftrightarrow|f\rangle\right.$ is forbidden by selection rules). The dissipative processes, like photon decay from the cavity or population decay from the excited states as well as ground state dephasing, are included into the description using a master equation. For computational reasons, we further restrict the number of Fock states of the cavity field to three (corresponding to photon numbers $n_{\text {photon }}=0,1,2$ ). As the dimension of the Hilbert space scales exponentially with the number of atoms $N$, only the one and two atom cases have been calculated numerically. This model [Fig. 3(b)] fits the data with the same set of parameters $\left(g, \Omega_{\text {con }}, \gamma_{\text {deph }}\right) / 2 \pi=(3.0,2.8,0.15) \mathrm{MHz}$ for one and two atoms, except for an independently measured, additional differential light shift of approx. $100 \mathrm{kHz}$ originating mainly from the control laser. The effective value of $g$ arises mainly from the distribution over Zeeman substates but also from motional averaging.

So far, only the dynamics of the internal states has 
been discussed. However, the interaction of atoms with near-resonant light fields is also strongly connected with their motional state due to the exchange of photon momenta. The interaction of two-level atoms with the cavity field can induce strong cooling forces 21, leading to, e.g. long storage times in experiments [22]. Cavity cooling is probably of relevance also for our system 18 . For threelevel atoms, the creation of a transparency window close to an absorption peak much narrower than the atomic line width by EIT can give rise to another sub-Doppler cooling mechanism [23], as has been demonstrated with trapped ions [24, 25]. In our experiment, strong cooling and heating effects close to the two-photon resonance have been observed. We have measured the probability of one atom to remain trapped inside the cavity for a time interval of $20 \mathrm{~ms}$ and $300 \mathrm{~ms}$, respectively, for different values of the two-photon detuning $\delta$ and have compared it with the survival probabilities for large two-photon detunings, see Fig. 4(a). We have observed a cooling region at $\delta=0$ in between a narrow weak and a strong broad asymmetric heating region at $\delta / 2 \pi=-0.2 \mathrm{MHz}$ and $\delta / 2 \pi=0.3 \mathrm{MHz}$, respectively. Without cooling, i.e. only with the far-off resonant trapping lasers, the $1 / e$ lifetime of atoms inside the resonator is $(0.7 \pm 0.1) \mathrm{s}$, see Fig. 4(b). The cooling at $\delta=0$ extends the lifetime to $(16 \pm 3)$ s. Far from the two-photon-resonance, the lifetime is compatible with the value of the uncooled atoms. While a clean distinction between cavity and EIT-cooling effects would require an advanced theoretical model, we utilize this cooling mechanism in our experimental sequence for the measurement of the EIT spectrum. By alternating cooling intervals with measurement intervals, we are able to increase the number of measurement cycles per atom by about a factor of 5 . The cooling mechanism could be further employed to obtain a stronger and more stable coupling of single particles to a cavity as is desired for many protocols in quantum information processing.

Our demonstration of optical control of the optical properties of a single atom has numerous implications for quantum engineering with single atoms: While linear absorption is suppressed due to destructive interference in EIT, nonlinear susceptibilities, giving rise to frequency summing and parametric amplification, can be resonantly enhanced [1, 26]. Furthermore, nonlinearities lead to an effective interaction between single photons. This is the basis of quantum logic gates [13, 27. However, in our case, still many photons are needed in the control laser beam to change the optical properties of the atom. Using a second cavity mode to enhance the interaction with the control beam could lead to an optical transistor for single photons [28, 29].

We would like to thank Lingbo Kong and Jonathan Simon for early ideas. We acknowledge financial support by the EC through AQUTE. R.R. and T.K acknowledge support from the Studienstiftung des deutschen Volkes and the Bonn-Cologne Graduate School of Physics and
Astronomy.

kampschulte@iap.uni-bonn.de

[1] S. E. Harris, J. E. Field, and A. Imamoglu, Phys. Rev. Lett., 64, 1107 (1990)

[2] M. Fleischhauer, A. Imamoglu, and J. P. Marangos, Rev. Mod. Phys., 77, 633 (2005)

[3] S. E. Harris, J. E. Field, and A. Kasapi, Phys. Rev. A, 46, R29 (1992)

[4] M. Xiao, Y.-q. Li, S.-z. Jin, and J. Gea-Banacloche, Phys. Rev. Lett., 74, 666 (1995).

[5] O. Schmidt, R. Wynands, Z. Hussein, and D. Meschede, Phys. Rev. A, 53, R27 (1996)

[6] L. V. Hau, S. E. Harris, Z. Dutton, and C. H. Behroozi, Nature, 397, 594 (1999).

[7] M. Fleischhauer and M. D. Lukin, Phys. Rev. Lett., 84, 5094 (2000).

[8] J. J. Hopfield, Phys. Rev., 112, 1555 (1958).

[9] D. F. Phillips, A. Fleischhauer, A. Mair, R. L. Walsworth, and M. D. Lukin, Phys. Rev. Lett., 86, 783 (2001)

[10] C. Liu, Z. Dutton, C. Behroozi, and L. Hau, Nature, 409, $490(2001)$

[11] U. Schnorrberger, J. D. Thompson, S. Trotzky, R. Pugatch, N. Davidson, S. Kuhr, and I. Bloch, Phys. Rev. Lett., 103, 033003 (2009).

[12] A. D. Boozer, A. Boca, R. Miller, T. E. Northup, and H. J. Kimble, Phys. Rev. Lett., 98, 193601 (2007)

[13] Q. A. Turchette, C. J. Hood, W. Lange, H. Mabuchi, and H. J. Kimble, Phys. Rev. Lett., 75, 4710 (1995)

[14] L. Slodička, G. Hétet, S. Gerber, M. Hennrich, and R. Blatt, Phys. Rev. Lett., 105, 153604 (2010)

[15] W. R. Kelly, Z. Dutton, J. Schlafer, B. Mookerji, T. A. Ohki, J. S. Kline, and D. P. Pappas, Phys. Rev. Lett., 104, 163601 (2010)

[16] A. A. Abdumalikov, O. Astafiev, A. M. Zagoskin, Y. A. Pashkin, Y. Nakamura, and J. S. Tsai, Phys. Rev. Lett., 104, 193601 (2010).

[17] M. Mücke, E. Figueroa, J. Bochmann, C. Hahn, K. Murr, S. Ritter, C. J. Villas-Boas, and G. Rempe, Nature, 465, 755 (2010).

[18] M. Khudaverdyan, W. Alt, I. Dotsenko, T. Kampschulte, K. Lenhard, A. Rauschenbeutel, S. Reick, K. Schörner, A. Widera, and D. Meschede, New J. Phys., 10, 073023 (2008)

[19] Y. Miroshnychenko, D. Schrader, S. Kuhr, W. Alt, I. Dotsenko, M. Khudaverdyan, A. Rauschenbeutel, and D. Meschede, Opt. Express, 11, 3498 (2003).

[20] The $25 \mathrm{kHz}$ linewidth is also the scattering rate of the control light for atoms in state $\left|g_{1}\right\rangle$.

[21] P. Domokos and H. Ritsch, J. Opt. Soc. Am. B, 20, 1098 (2003)

[22] S. Nußmann, K. Murr, M. Hijlkema, B. Weber, A. Kuhn, and G. Rempe, Nature Phys., 1, 122 (2005).

[23] G. Morigi, J. Eschner, and C. H. Keitel, Phys. Rev. Lett., 85, $4458(2000)$.

[24] C. F. Roos, D. Leibfried, A. Mundt, F. Schmidt-Kaler, J. Eschner, and R. Blatt, Phys. Rev. Lett., 85, 5547 (2000)

[25] F. Schmidt-Kaler, J. Eschner, G. Morigi, C. Roos, 
D. Leibfried, A. Mundt, and R. Blatt, Appl. Phys. B: Lasers Opt., 73, 807 (2001).

[26] M. Yan, E. G. Rickey, and Y. Zhu, Opt. Lett., 26, 548 (2001)

[27] L.-M. Duan and H. J. Kimble, Phys. Rev. Lett., 92, $127902(2004)$
[28] M. Soljacic, E. Lidorikis, J. D. Joannopoulos, and L. V. Hau, Appl. Phys. Lett., 86, 171101 (2005)

[29] P. Bermel, A. Rodriguez, S. G. Johnson, J. D. Joannopoulos, and M. Soljacic, Phys. Rev. A, 74, 043818 (2006) 